\title{
Bilateral posterior fracture-dislocation of the shoulders with subscapularis rupture and HAGL lesion: a case report
}

\author{
Wei REN ${ }^{1,3}$, Masumi MARUO HOLLEDGE ${ }^{1}$, Yongcheng $\mathrm{HU}^{2,3}$ and Jike LU ${ }^{1 *}$ \\ ${ }^{1}$ Department of Orthopedic Surgery, United FamilyHospital, No.2 Jiangtai Lu, Chaoyang District, Beijing, China \\ ${ }^{2}$ Department of Foot and Ankle Surgery, Tianjin Hospital, No. 406, Jie Fang Nan Lu, He Xi District, Tianjin, China \\ ${ }^{3}$ Tianjin Medical University, No.22, Qi Xiang Tai Lu, He Ping District, Tianjin, China
}

\begin{abstract}
Background: Bilateral posterior fracture-dislocation of the shoulders is a rare condition and easy to be overlooked. They can be caused by epileptic seizures, sports injuries, electrical shock, or electroconvulsive therap. We report a rare case of posterior fracture-dislocation of bilateral shoulders with the HAGL (Humeral Avulsion Glenohumeral Ligament) lesion on one shoulder, and subscapularis rupture on the other shoulder during a seizure attack in epileptics.

Case presentation: A 69-year-old male was admitted to our hospital two days after an epileptic seizure. Clinical examination showed severe pain and limited range of motion in both shoulders. The shoulder radiograph examination revealed the great tuberosity fractures and 'light bulb sign' bilaterally. CT examination showed glenohumeral joint fractures and posterior dislocations in bilateral shoulders. After immediate close reduction, MRI was performed. This showed comminuted fractures of the both humeral heads and necks, reverse Hill-Sachs lesions, complete subscapularis rupture on the left shoulder, and HAGL lesion on the right shoulder. We performed open reduction internal fixation of fractures, repair of subscapularis, and repair of HAGL lesion. At six month, the patient showed excellent outcome.

Conclusion: We treated successfully bilateral posterior fracture-dislocation of shoulders with subscapularis rupture and HAGL lesion after an epileptic seizure episode. As far as we know, this is the first report of this complicated condition. Not only physical examination and radiographic evaluation are important, but also MRI exam to investigating soft tissue injuries was one of the key points of this case. Open reduction internal fixation of fractures and reconstruction of ligaments followed by a rehabilitation program to address shoulder instability lead to a successful result.
\end{abstract}

\section{Introduction}

The shoulder is one of the most unstable joints accounting for nearly $50 \%$ of all dislocations [1]. However, the incidence of bilateral glenohumeral joint dislocation is rare. When a bilateral posterior dislocation is present, it is almost always posterior, and secondary to seizure activity. It can be also caused by sports injuries, electrical shock, or electroconvulsive therapy [2]. We report an unusual case of bilateral posterior fracture-dislocation of the glenohumeral joints with bilateral fractures of greater tuberosities and lesion of the inferior glenohumeral ligament (The HAGL lesion) on one shoulder and subscapularis compete rupture on the other shoulder.

\section{Case report}

A 69-year-old Canadian male complained bilateral shoulders pain, deformity, and unable to mobilize after a seizures attack two days prior to come to our hospital. The patient had no trauma history of shoulders nor seizures history. This was his first seizure attack and his wife witnessed that during the seizures attack, his shoulder was in abduction position with internal rotation, due to muscle contracture. His wife also heard cracking sound, although the patient could not recall the event. He went to a local hospital, took radiographs showing bilateral shoulder dislocations and humeral head fractures (Figure1) and he was transferred to us. Physical examination of bilateral shoulders showed swollen shoulder joints, skin bruises in anterior part, flattening of normal contour, palpable hollowness below the acromion processes, bulging at posterior shoulders, and tenderness both anterior and posterior sides. The patient was unable to do any shoulder movements due to pain.

The radiographs showed the humerus heads were rotated with great tuberosity fractures bilaterally. Although humerus heads were dislocated posteriorly, the both heads of humerus stayed in the same level with glenoid, showing so called 'light bulb sign'. The bilateral shoulder dislocation was confirmed by shoulder CT scans, showing bilateral glenohumeral joints posterior dislocation, with the humeral heads shift posteriorly and laterally as well as comminuted fractures on bilateral greater tuberosities and lesser tuberosities displacement. Also, the surrounding soft tissues in bilateral shoulders showed obvious swelling (Figure 2).

The patient was taken to operation theater for close reduction under general anesthesia. The post-operation radiographs confirmed the dislocation was relocated.

Then we performed MRI examinations on bilateral shoulders. MRI images revealed not only comminuted fractures of the humeral heads

Correspondence to: Jike LU, Department of Orthopedic Surgery, United FamilyHospital, No.2 Jiangtai Lu, Chaoyang District, Beijing, China, E-mail: jike.lu@ufh.com.cn

Key words: bilateral posterior fracture-dislocation of the shoulders, epileptic seizure, subscapularis, HAGL lesion

Received: February 24, 2017; Accepted: March 17, 2017; Published: March 22, 2017 

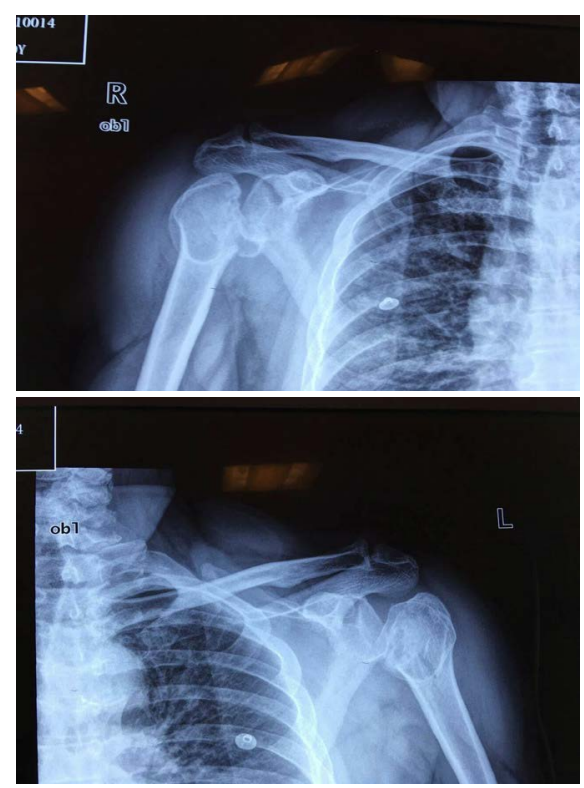

Figure 1. The radiographs shows the humerus heads are rotated with great tuberosity fractures bilaterally. Although humerus heads were dislocated posteriorly, the both heads of humerus stayed in the same level with glenoid, showing so called 'light bulb sign'.
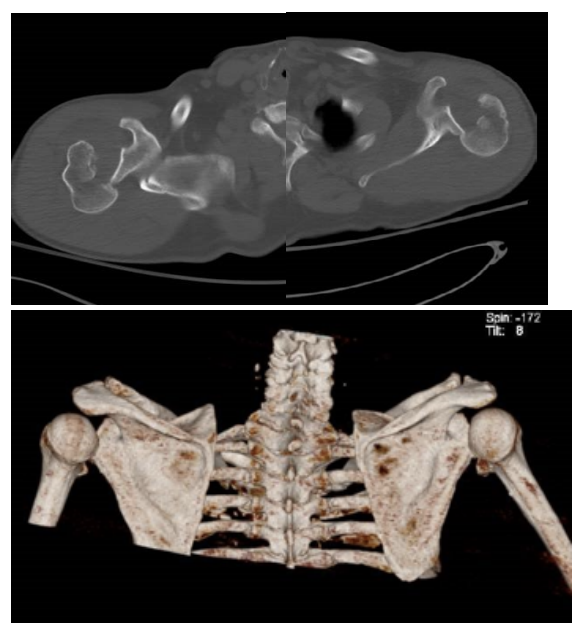

Figure 2. Bilateral shoulder CT scans are showing bilateral glenohumeral joints posterior dislocation. The humeral heads shift posteriorly and laterally as well as comminuted fractures on bilateral greater tuberosities and lesser tuberosities displacedment. Also surrounding soft tissues in bilateral shoulders showed obvious swelling.

and necks and reverse Hill-Sachs lesion, also HAGL lesion on the right shoulder (Figure 3a) and complete rupture of subscapularis tendon on the left shoulder (Figure $3 b$ ).

Two days later, the patient had left shoulder surgery. Using the standard deltopectoral approach, clavipectoral fascia was dissected, we found a completely ruptured subscapularis tendon at the glenoid side. Following the anterior capsulotomy, a T-shape cut was made to expose the glenoid. There was no significant labrum tear. The antero-medial humeral head impression fracture was elevated with a periosteum elevator, lesser tuberosity was reattached to its footprint, and we fixed this with four cannulated screws. Another two cannulated screws were used for greater tuberosity fracture fixation. We filled bone marrow depression void with 10 grams of allograft granules. The allograft was impacted firmly and periosteum was sutured back. The inferior-medial capsule was sutured back to superior-lateral aspect of the capsule (capsulorrhaphy). The torn subscapularis tendon was repaired with two bone anchors and reinforced with fiber wires (Figure 4a).

Six days after injury, the patient received right shoulder surgery. We experienced similar procedures: the less tuberosity was re-attached to its footprint with three cannulated screws, except there was no great tuberosity fracture on right side. In addition, there was a HAGL lesion; the complete avulsion of glenohumeral ligament with a piece of bone fragment was re-attached to humeral foot print with a $2.9 \mathrm{~mm}$ bone anchor, reinforced with fiber wires (Figure $4 \mathrm{~b}$ ).

Post operatively, both shoulder was immobilized with shoulder immobilizers for one week. At second week, the patient started passive shoulder movements, and at six week, the patient started active movements. At six months, the patient had a free of pain and regain full active range of motion bilaterally (Figure 5).

\section{Discussion}

Bilateral dislocation of the shoulders is a rare condition, accounting for 0.6 per100,000 of shoulder dislocations in general population [1]. Bilateral posterior shoulder dislocations are even rarer as it is accounting for $5-15 \%$ of all posterior dislocations. The causes are mainly associated with seizure attacks, and the other reasons are including high energy sports injuries, electrical shock, electroconvulsive therapy,

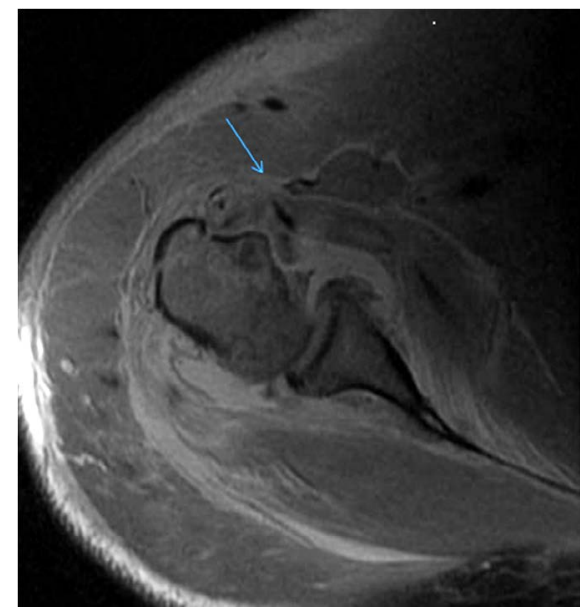

Figure 3a. MRI images shows anterior avulsion of the inferior glenohumeral ligament from the humeral neck of the left shoulder (HAGL lesion) (Arrow)

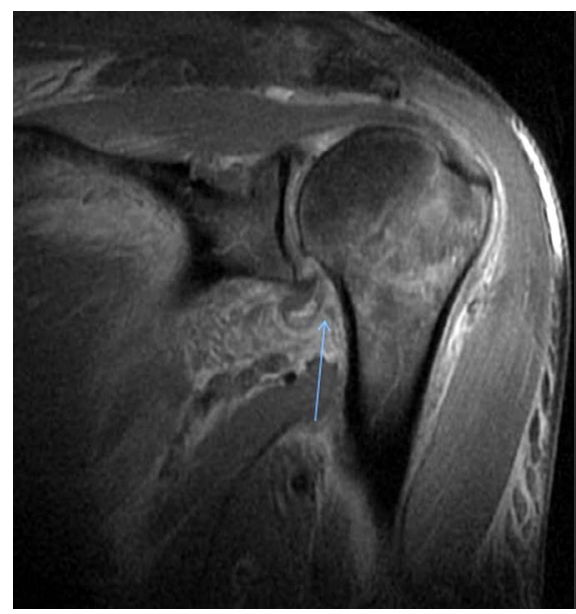

Figure 3b. MRI shows the subscapularis tendon rupture with small piece of bone of the right shouder (Arrow). 


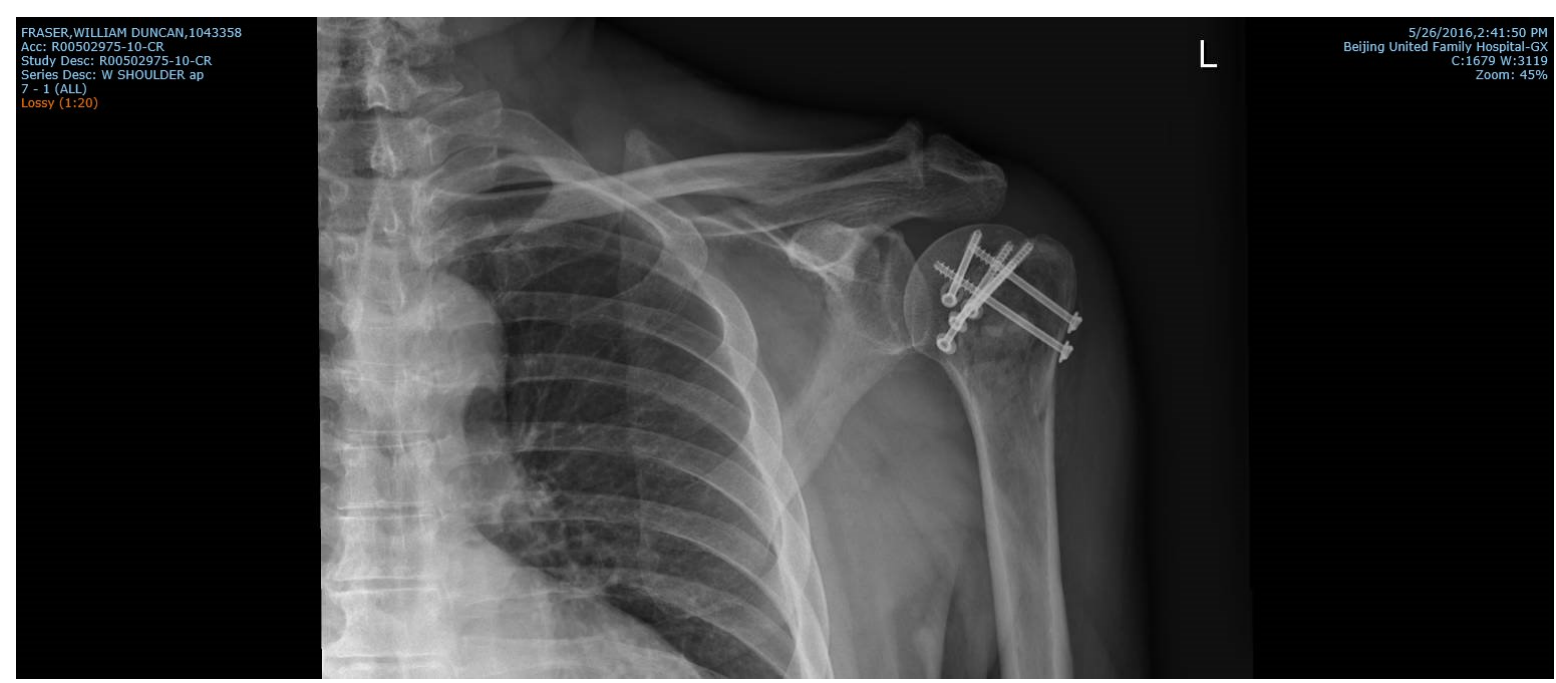

Figure 4a. Post-operative radiograph of left shoulder

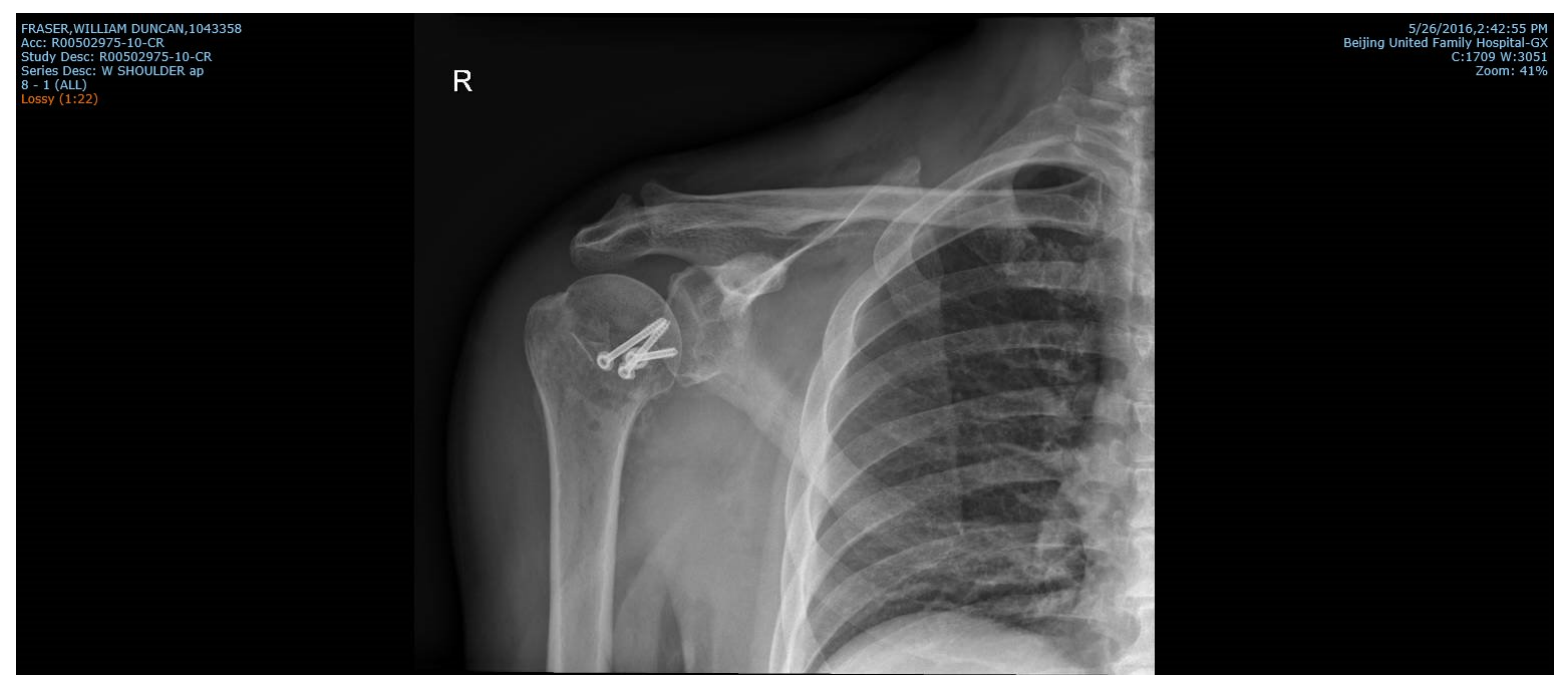

Figure 4b. Post-operative radiograph of right shoulder

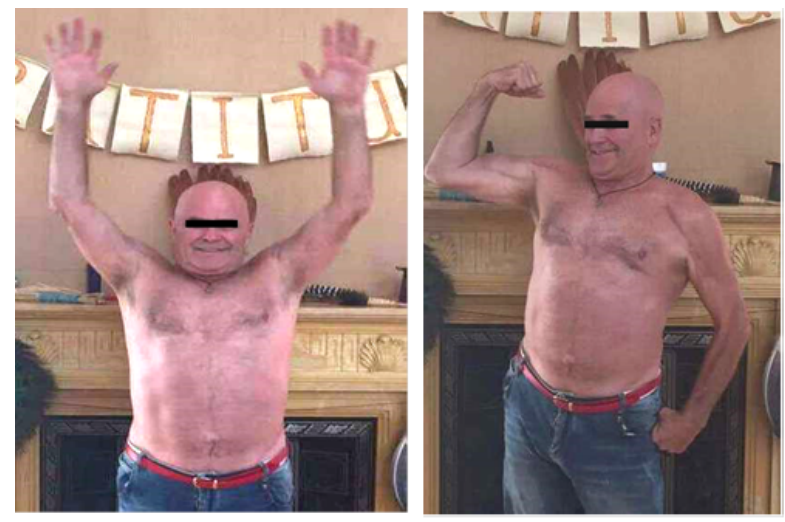

Figure 5. At six months, post-operation

hypoglycemic episodes and dyskinetic disorders [2]. The biophysical basis of posterior dislocation is believed to be a violent involuntary contraction of muscles around the shoulder, especially the internal rotators and the abductors, which are stronger in comparison to the external rotators and adductors [3]. These muscles pull and rupture the muscle, tendon, labrum, and capsule of the shoulder joint causing shoulder dislocations posteriorly [3].

A major threat to this condition is a missed diagnosis. The literature estimates that the diagnosis of posterior glenohumeral dislocation is missed in approximately $60 \%$ to $79 \%$ of cases [4]. This results in delaying the treatment of the dislocation and increasing risk of more complications [4]. At first, patients will complain pain and limitation of movement of the shoulder. The condition of limited active and passive range of motion often leads to incorrect diagnosis including frozen shoulders. In the AP view radiographs, unlike anterior shoulder dislocations which will show the shift of the head of humerus to inferiorly, the head of humerus will stay in the same level as glenoid for the posterior glenohumeral dislocations. However, there is a light bulb sign' which can indicate posterior dislocation. This sign is due to the humeral head rotates internally and this is producing the more round and symmetric shape in the setting of a posterior dislocation.

The posterior shoulder dislocations are often associated with a reverse Hill-Sachs lesion, which is an impression fracture of the anterior aspect of the humeral head [5]. In addition, fractures of the posterior aspect of the glenoid rim, fractures of greater or lesser tuberosities, 
tears of capsulo-labral complex or rotator cuff may also accompany in severe cases [6]. Treating the reverse Hill-Sachs deformities is crucial in the posterior dislocation especially for chronic cases. McLaughlin [7] recommended transfer of the subscapularis tendon into the defect in order to prevent recurrence of glenohumeral instability. Hawkins et al. [8] modified the McLaughlin technique suggesting the transfer of the lesser tuberosity with the attached subscapularis tendon for more secure fixation of the tendon into the defect, using suture anchors rather than detachment and reattachment of the tendon into the defect. The treatment strategy has to consider conditions such as the duration of dislocation, the size of the humeral head defect, the presence of fracture and on the patient's age, health and functional status [6]. The size of the defect is the decisive criterion in determining the recommended treatment method $[9,10]$. Cicak [10] recommended non-operative treatment for minor reverse Hill-Sachs lesions (affecting less than $25 \%$ of the humeral articular surface). Up to $25 \%$ defects of humeral head articular surface can be treated by closed or open reduction only, especially in acute cases (less than three weeks) closed reduction should be attempted first.

Humeral head reconstruction can be achieved by filling the defect with allograft or autograft with/without osteosynthesis to stabilize or underlay the restored surface [11]. Many authors have advocated the modified McLaughlin technique for the defects involving 30\% - 40\% of the humeral head $[12,13,14]$. Some arthroscopic techniques have been described to treat defects up to $30 \%$ [15]. For the defects of involving more than $40 \%$ of the articular surface, or the head shows osteonecrosis, or delayed diagnosis (more than six months), total/hemi-arthroplasty needs to be considered [16].

A displaced great tuberosity fracture usually signifies an associated rotator cuff injury. If this was not reduced anatomically, the injury can develop to permanent disability of significantly decreased range of motion of the shoulder joint. Open reduction and internal fixation is recommended if the great tuberosity fracture is greater than $5 \mathrm{~mm}$ displacement, or angulation is more than 45 degree, because these displacement conditions are caused by the pull of subscapularis tendon. A displaced great tuberosity fracture may lead to aggravation, malunion, impingement, or dislocation of the tendon of the long head of biceps.

HAGL is the result of acute traumatic glenohumeral subluxation or dislocation. Anterior avulsion of the inferior glenohumeral ligament (IGHL) from the humeral neck is the more common lesion. The IGHL complex is composed of the anterior band, posterior band, and axillary pouch [17]. The ligament complex serves as one of the important glenohumeral joint static stabilizers. HAGL lesion result in incompetence of the IGHL complex, which leads to instability of glenohumeral joint. MRI is one of the best investigation to diagnose the HAGL lesion. A U-shape axillary pouch can be recognized in MRI imagines and it appears a J-shape if HAGL lesion exists. The HAGL lesion rarely happens isolate, and usually associated with rotator cuff injuries or fractures. The HAGL lesion can be managed by arthroscopic repair or open re-attachment at the same time of an open surgery to fix fracture or rotator cuff. Excellent results have been achieved with open surgical management through subscapularis approach. Miniopen techniques involving limited incision in the lower one half of the subscapularis also can be done [18].

\section{Conclusion}

We present a rare case of bilateral posterior dislocation of the shoulders after a seizes attack. The case was involved with bilateral glenohumeral joint dislocations and great tuberosity fractures, with one side subscapularis rupture at lesser tuberosity, and the other side presented a HAGL lesion. Physical examination and radiographic evaluation are important in order to avoid miss-diagnosis, especially timely close reduction is needed. MRI investigation for soft tissue injuries will provide important information for surgical plan. We believe open reduction internal fixation of fractures and reconstruction of ligaments followed by a rehabilitation program to address shoulder instability will have a successful result.

\section{References}

1. Robinson CM, Akhtar A, Mitchell M, Beavis C (2007) Complex posterior fracturedislocation of the shoulder. Epidemiology, injury patterns, and results of operative treatment. J Bone Joint Surg Am 89: 1454-1466. [Crossref]

2. Ketenci IE, Duymus TM, Ulusoy A, Yanik HS, Mutlu S, et al. (2015) Bilateral posterior shoulder dislocation after electrical shock: A case report. Ann Med Surg (Lond) 4: 417 421. [Crossref]

3. Siu YC, Lui TH (2014) Bilateral anterior shoulder dislocation. Arch Trauma Res 3 : e18178. [Crossref]

4. Pourmand A, Marcozzi D, Shokoohi H, Alhmoudi A, Olafson, S (2016) Atraumatic bilateral posterior shoulder dislocations, a rare case of sleep deprivation. Am J Emerg Med 34: 1322 e1323-1324. [Crossref]

5. Provencher MT, Frank RM, Leclere LE, Metzger PD, Ryu JJ (2012) The Hill-Sachs lesion: diagnosis, classification, and management. J Am Acad Orthop Surg 20: 242 252. [Crossref]

6. Kowalsky MS, Levine WN (2008) Traumatic posterior glenohumeral dislocation classification, pathoanatomy, diagnosis, and treatment. Orthop Clin North Am 39: 519 533, viii. [Crossref]

7. Mc LH (1952) Posterior dislocation of the shoulder. J Bone Joint Surg Am 24: 584-590

8. Hawkins RJ, Neer CS 2nd, Pianta RM, Mendoza FX (1987) Locked posterior dislocation of the shoulder. J Bone Joint Surg Am 69: 9-18. [Crossref]

9. Paul J, Buchmann S, Beitzel K, Solovyova O, Imhoff AB (2011) Posterior Shoulder Dislocation: Systematic Review and Treatment Algorithm. Arthroscopy 27: 1562-1572. [Crossref]

10. Cicak N (2004) Posterior dislocation of the shoulder. J Bone Joint Surg Br 86: 324-332.

11. Begin M, Gagey O, Soubeyrand M (2012) Acute bilateral posterior dislocation of the shoulder: one-stage reconstruction of both humeral heads with cancellous autograft and cartilage preservation. Chir Main 31: 34-37. [Crossref]

12. Diklic ID, Ganic ZD, Blagojevic ZD, Nho SJ, Romeo (2010) Treatment of locked chronic posterior dislocation of the shoulder by reconstruction of the defect in the humeral head with an allograft. J Bone Joint Surg Br 92: 71-76.

13. Gavriilidis I, Magosch P, Lichtenberg S, Habermeyer P, Kircher, J (2010) Chronic locked posterior shoulder dislocation with severe head involvement. Int Orthop 34: 79-84. [Crossref]

14. Kokkalis ZT, Mavrogenis AF, Ballas EG, Papanastasiou J, Papagelopoulos PJ (2013) Modified McLaughlin technique for neglected locked posterior dislocation of the shoulder. Orthopedics 36: e912-916. [Crossref]

15. Engel T, Hepp P, Osterhoff G, Josten C (2009) Arthroscopic reduction and subchondral support of reverse Hill-Sachs lesions with a bioabsorbable interference screw. Arch Orthop Trauma Surg 129: 1103-1107. [Cros`sref]

16. Claro R, Sousa R, Massada M, Ramos J, M Lourenço J (2009) Bilateral posterior fracture-dislocation of the shoulder: Report of two cases. Int J Shoulder Surg 3: 41-45. [Crossref]

17. Burkart AC, Debski RE (2002) Anatomy and function of the glenohumeral ligaments in anterior shoulder instability. Clin Orthop Relat Res 32-39. [Crossref]

18. George MS, Khazzam M, Kuhn JE (2011) Humeral avulsion of glenohumera ligaments. J Am Acad Orthop Surg 19: 127-133. [Crossref]

Copyright: (C2017 Wei REN. This is an open-access article distributed under the terms of the Creative Commons Attribution License, which permits unrestricted use, distribution, and reproduction in any medium, provided the original author and source are credited. 